\title{
ESTRESSE FISIOLÓGICO DO ARBITRO DE BASQUETEBOL: REVISÃO SISTEMÁTICA
}

\section{ARTIGO DE REVISÃO}

INCHAUSPE, Ramiro Marques ${ }^{1}$

BARBIAN, Pablo Morales ${ }^{2}$

INCHAUSPE, Ramiro Marques. BARBIAN, Pablo Morales. Estresse fisiológico do arbitro de basquetebol: Revisão sistemática. Revista Científica Multidisciplinar Núcleo do Conhecimento. Ano 04, Ed. 07, Vol. 13, pp. 20-45. Julho de 2019. ISSN: 2448-0959

\section{RESUMO}

Objetivos: A presente revisão fornece um resumo das atividades de jogo dos árbitros de basquete, avaliação de aptidão física, função cognitiva e gasto de energia (GE) durante os jogos oficiais. Métodos: Analisando a literatura sobre árbitros de basquete. Revisão bibliográfica realizada utilizando Pub Med, WOK, SCOPUS e Google Scholar, com critérios de busca incluindo os termos "árbitros", "oficiais de jogo", "basquete" e termos de desempenho como "fisiológicos", "demandas físicas", "tomada de decisão" e "fadiga mental". Resultados: os estudos relevantes $(n=19)$ mostraram que os árbitros cobrem $5.000 \mathrm{~m}$ durante um jogo com frequências cardíacas (FC) médias de 140 bat.min-1 (> 75\% FCmax) e consumo de oxigênio (VO2) de $52 \mathrm{ml}$.min1.kg-1 ( 86,19\% VO2max). O desempenho do teste de recuperação intermitente YO-

${ }_{1}^{1}$ Fisioterapeuta e Educador Físico, Mestre e Doutorando, Coordenador de Ciência e Aptidão Física da Federação Internacional de Basketball (FIBA), Coordenador de Aptidão Física da Confederação Brasileira de Basketball (CBB), Professor Universitário e Pôs Graduação Fisiowork/Redentor, PUC/RS, Uniritter.

2 Fisioterapeuta, Coordenador de Aptidão Física da Confederação Brasileira de Basketball (CBB), Treinador $\mathrm{CORE} 360^{\circ}$, Professor de pós graduação em Traumatologia e Ortopedia Aplicada a Reabilitação na Empresa Fisiowrk/Redentor, Sócio Fundador da Empresa Funcional Poa Cursos. 
YO nível 1 (YO-YO-IRT1) revelou uma validade direta para avaliar a atividade aeróbica intermitente, uma vez que estava correlacionada com a atividade em campo dos árbitros. Os árbitros estimados de GE durante um jogo excedem $500 \mathrm{kcal}$ ( 5 kcal.kg-1.h-1). O limiar de baixa intensidade e alta intensidade pode ser considerado menor que 3,8 MET e maior que 9,8 METs, respectivamente. As habilidades físicas dos árbitros diminuem gradualmente com a idade cronológica. No entanto, os árbitros mais velhos (> 32 anos) têm uma qualidade significativamente melhor do que os árbitros jovens. As demandas cognitivas-perceptivas da arbitragem do basquetebol também são discutidas na presente revisão. Conclusão: A análise do jogo dos árbitros de basquete mostrou uma atividade intermitente (proporção de 1: 12 de esforço / recuperação de alta intensidade). O YO-YO-IRT1 poderia ser usado como um teste de aptidão específico para estimar sua capacidade aeróbica. A GE dos árbitros mostrou uma "intensidade energética moderada" quando expressa por equivalente metabólico ( 5 METs). Um programa de treinamento específico deve ser prescrito para os árbitros, levando em consideração sua idade. Os processos cognitivos que influenciam as decisões dos árbitros de basquete devem ser estudados em outras pesquisas.

Palavras-chave: análise de jogos, teste físico, performance, árbitros basquetebol.

\section{INTRODUÇÃO}

A arbitragem é, sem dúvida, um dos aspectos mais polêmicos envolvidos na competição esportiva, sendo frequentemente citada por atletas, treinadores e dirigentes como responsáveis por seus insucessos e fonte de estresse. Este fato pode ser explicado pelo potencial conflito de interesses entre dois pólos: um que envolve o que a arbitragem viu e decidiu no lance em função do seu ângulo de observação, momento emocional e preparo físico, e outro que envolve o que os demais interessados (atletas, comissão técnica, dirigentes, torcedores) conseguiram ver dos seus respectivos locais de observação. Estas diferenças de ponto de vista e tomadas de decisão acabam desencadeando uma série de reações estressantes dentro do meio ambiente esportivo $[1,38,50]$.

Assim, todos os fatores e capacidade que dizem respeito a preparação árbitro de basquetebol pode influenciar nas tomadas de decisão durante uma partida, RC: 34836

Disponível em: https://www.nucleodoconhecimento.com.br/educacao-fisica/estresse-fisiologico 
preparação psicológica e mental, preparação emocional e preparação física, pois quanto mais preparado de forma geral o árbitro estiver, maior será a chance de o mesmo estar melhor posicionado, mais próximo, estar bem fisicamente irá proporcionar uma melhor tomada de decisão pois sua mente estará descansada para melhor avaliar as jogadas, quando houver lances decisivos e também saber tomar a decisão correta em momentos de pressão. Portanto, para conduzir as partidas de nível profissional, o árbitro deve apresentar um ótimo nível de preparação psicológica e física, pois seu esforço mental e físico é semelhante ao dos jogadores [1, 38, 50].

Os árbitros de basquetebol estão inseridos no contexto da Federação Internacional de Basketball (FIBA), que organiza e regula o esporte em nível mundial, dentre os requisitos impostos pela mesma para o árbitro estar apito a participar dos jogos deve se submeter e ter êxito em exame teórico sobre regras e situações do jogo assim como teste de aptidão física.

Conforme demonstrado em diversos estudos é possível constatar a importância da arbitragem no âmbito esportivo e principalmente no basquetebol, bem como a adesão de programas de treinamento mental, comportamental e preparação física para a melhor performance dos árbitros [38].

\section{MÉTODOS}

\section{CRITÉRIOS DE INCLUSÃO / EXCLUSÃO}

Os estudos foram considerados elegíveis para inclusão se satisfizessem os seguintes critérios: (1) foram um estudo experimental publicado em uma revista arbitrada em inglês; (2) usaram participantes humanos sem doença ou lesão crônica e (3) a descrição de pelo menos um dos principais resultados (por exemplo, demandas físicas, fisiológicas, mentais, envelhecimento, testes de condicionamento físico ...). Os estudos foram excluídos se: (1) Não atenderem aos requisitos mínimos quanto à descrição das variáveis de desempenho relacionadas entre os árbitros de basquete; (2) Não relatou resultados adequadamente (média e desvio padrão); (3) Não relatou resultados sobre árbitros de basquete e (4) a descrição das variáveis psicológicas.

\section{ESTRATÉGIA DE BUSCA}


Uma revisão da literatura disponível sobre Pub Med., SCOPUS e Google Scholar envolvendo uma sintaxe de pesquisa booleana das seguintes palavras-chave: 'árbitros' ou 'oficiais de jogo' e 'basquete' e 'fisiológicos' e 'demandas físicas' ou ' tomada de decisão 'e' fadiga mental 'foi realizada. Além disso, uma pesquisa manual foi usada por meio das listas de referência dos artigos abrangidos. Não houve data limite para o período de pesquisa (ou seja, os estudos que foram publicados antes de dezembro de 2018) e apenas estudos publicados em inglês foram examinados. Para organizar os resultados, o os achados do estudo foram agrupados de acordo com o sistema do árbitro (três e / ou dois), o nível de competição (nacional ou internacional) e as categorias de jogadores (sênior, U-19 e masculino, ou feminino).

\section{RESULTADOS}

Dezenove estudos são incluídos de 416 considerados; os resultados são apresentados juntamente com os seis principais resultados dos árbitros durante 0 jogo: respostas fisiológicas, atividades físicas, envelhecimento e desempenho físico, função cognitiva, fadiga e avaliações de fitness. Após triagem e filtro, doze estudos foram incluídos em um total de 25 artigos nas bases de dados Pub Med., WOK e SCOPUS, e 7 estudos foram selecionados de um total de 249 no Google Scholar e na busca manual.

Os diferentes estudos relataram que a média da FC dos árbitros de basquetebol variou de 130 a 174 batimentos.min-1 (ou seja, 70 a 96\% da FCmax). Durante os jogos nacionais, a média de FC dos árbitros de basquetebol foi relativamente menor do que os valores registrados durante os jogos internacionais.

A capacidade aeróbia, expressa como consumo máximo estimado de oxigênio (VO2max), dos árbitros de basquetebol de diferentes ligas (competição sul-americana, europeia, australiana e africana). A média do VO2máx variou de 41,42 a 58,29 (ml.kg.1.min-1). A maior média de VO2max foi observada entre os árbitros de basquete croatas, que participaram da $1^{\underline{a}}$ Liga de Basquete 2008/2009 (52,49 \pm 5,8 ml.kg.-1.min1).

A GE estimada dos árbitros de basquetebol foi resumida de acordo com o período do jogo e o sistema de arbitragem (ou seja, dois ou três árbitros por jogo). Os jogos 
oficializados com dois árbitros mostraram uma carga de energia calórica significativamente maior durante 0 quarto dia do que no $1^{\circ}$ dia $(p=0,007)$. A competição envolvendo dois e três árbitros por jogo mostrou que a distância percorrida total média variou de 3059 a 6773 me 3260 a 6440 m, respectivamente. Os resultados da análise de tempo e movimento do árbitro durante os jogos oficiais apresentaram como porcentagem o tempo total gasto em cada categoria de movimento. Independentemente do sistema de arbitragem durante os jogos, a porcentagem do tempo total gasto em atividades de baixa intensidade (ou seja, em pé, andando e jogging) parecia ser maior do que aquela gasta em atividades de alta intensidade. Os jogos oficializados pelo sistema dos dois árbitros, mostraram que uma porcentagem maior do tempo total em atividades de alta intensidade (ou seja, corrida e corrida) do que os jogos jogados com o sistema de três árbitros.

Os diferentes desempenhos dos árbitros de basquete em relação à sua idade (ou seja, "mais jovens" e "mais velhos"). Todos os desempenhos físicos mostraram uma diferença significativa $(p<0,05)$ entre árbitros mais jovens mais velhos. No entanto, nenhuma diferença significativa foi relatada na capacidade aeróbica (ou seja, VO2max).

\section{DISCUSSÃO}

O monitoramento da frequência cardíaca $(F C)$ tem sido usado como um indicador da tensão fisiológica durante o jogo [13]. Nesse contexto, os estudos tiraram conclusões diferentes, quando os resultados da $\mathrm{FC}$ do árbitro foram comparados entre quartos de jogo ( $Q$ ) e metades. De facto, cada jogo (isto é, regras da FIBA) consiste em quatro $Q$ de 10 minutos com um intervalo de descanso de 15 minutos no intervalo (entre Q1 + $\mathrm{Q} 2$ e Q3 + Q4). Apenas uma pausa de 2 minutos é dada entre o $Q$ de cada metade [2]. Vaquera et al. [5] relataram que a FC média do jogo foi significativamente menor durante o $4^{\circ} Q$ em relação ao $2^{\circ} Q(p<0,001)$ e significativamente menor no $3^{\circ}$ comparado ao $2^{\circ} Q(p=0,005)$ entre árbitras de basquetebol feminino durante jogos internacionais. Durante o Campeonato Eurobasket masculino de 2013, Vaquera et al. $[5,6]$ constataram que a FC média do jogo foi significativamente maior no primeiro tempo do que no segundo tempo (163,5 $\pm 14,8$ vs $158,45 \pm 19,9$ bat.min-1, respectivamente). Isso mostrou a semelhança da intensidade relativa do jogo, 
independentemente do jogo (Campeonato Eurobasket masculino x feminino), usando o sistema dos três árbitros por jogo. Considerando que Borin et al. [3] encontraram diferenças significativas na média de FC dos árbitros de basquete, quando compararam o $Q$ do jogo na semifinal e nas fases finais do Campeonato Nacional de Basquete do Brasil 2009-2010 para adultos do sexo masculino. Este resultado foi o oposto do que foi observado nos jogos da fase de qualificação [3]. Estes sugerem que a tensão relativa imposta aos árbitros de basquetebol (isto é, o sistema de três árbitros por jogo) diminui quando o nível de competição é menor. Estes achados poderiam ser apoiados pelas pesquisas de Leicht [14,15], quando a média do árbitro central durante os jogos nacionais e internacionais foi comparada (73 vs 78,7\% da FCmax).

Vaquera et al. [6] compararam árbitros masculinos e femininos durante um nível semelhante de competição e mostraram que as árbitras femininas exibiam uma média de FC menor $(156,8 \pm 10,2$ vs 163,6 \pm 11,6 bat.min-1, $p<0,05)$ e intensidade do exercício (86,2 $\pm 5,5$ vs. $89,5 \pm 6,0 \%$ FCmax, $p<0,05)$ que os do sexo masculino, o que sugere que o sexo influencia significativamente as respostas cardiovasculares na arbitragem de basquete.

A tensão cardiovascular parece ser acentuada em campeonatos internacionais. No entanto, o estresse cardiovascular pode ser menor na competição nacional em comparação com um torneio internacional, quando, obviamente, considerando jogos que aplicaram o sistema do mesmo árbitro. Por exemplo, Leicht [15] relatou um jogo de FC médio (78,7 \pm 8,8\% da FCmax) que é ligeiramente superior aos valores (74,9 \pm $6,8 \%$ da FCmax) relatados por Nabli et al. [16] durante uma competição internacional e nacional, respectivamente. Por outro lado, sabendo que os procedimentos de arbitragem dos jogos foram os da FIBA, com dois árbitros por jogo, no Nabli et al. [2] e Leicht [15] mostraram que não houve diferenças significativas entre o jogo $Q$ e as metades, mesmo quando as taxas de trabalho diminuíram no final do jogo. Essa consistência na FC durante todo o jogo, acompanhada de uma taxa de trabalho de drop-in, foi atribuída à deriva cardiovascular que ocorre com o exercício prolongado [17]. Na verdade, os árbitros são obrigados a "seguir" o jogo, movendo-se em posições estratégicas para julgar as ações dos jogadores até os últimos momentos do jogo. Além disso, a idade dos árbitros e as diferenças na natureza de suas tarefas quando comparados aos jogadores também devem ser levados em consideração [2]. As 
experiências do árbitro demonstraram influenciar significativamente a sua FC. De fato, Vaquera et al. [5] identificaram uma correlação entre a FC média do jogo e a experiência das árbitras $(r=-0,653, p=0,008)$ durante a competição internacional de elite (ou seja, três árbitros por jogo). Da mesma forma, Nabli et al. [7] também observaram uma correlação significativa entre a experiência de árbitros masculinos e a FC do jogo $(r=-0,826, p=0,001)$ no campeonato de basquetebol sub-19 da Tunísia.

Em relação à capacidade aeróbica, considerando que esta revisão tem enfatizado o VO2max estimado de árbitros de basquetebol usando equações válidas em diferentes testes de aptidão em campo ou em laboratório. Os estudos mostraram uma capacidade boa a excelente para os níveis nacional e internacional, respectivamente, levando em conta os critérios de classificação do VO2máx propostos pela American Heart Association [18] para não atletas. De fato, Rupčić et al. [19] relataram que os árbitros croatas classificam principalmente em categorias de muito boas a excelentes capacidades aeróbicas [20], quando comparadas com a população inexperiente na Croácia. Em relação ao nível dos árbitros, Bonganha et al. [21] relataram uma diferença não significativa entre árbitros nacionais e internacionais $(47,07 \pm 5,22$ vs 45,35 \pm 4,22 ml.kg.-1.min-1, $p=0,321$, respectivamente). No mesmo contexto, Rupčić et al. [19] mostraram uma diferença não significativa entre árbitros de basquete mais jovens e mais velhos (54,04 vs 50,84 ml.kg.-1.min-1, $p=0,127$, respectivamente). Isso pode sugerir que a capacidade aeróbica poderia ser relativamente similar entre os árbitros de basquete, independentemente de seu nível de arbitragem e idade, o que os ajuda a acompanhar o jogo.

A FC tem sido utilizada em conjunto com equações de regressão, determinando a relação entre a FC e o VO2 (derivado de avaliações laboratoriais), para estimar GE [16]. Apesar das mudanças irregulares na intensidade do exercício durante o jogo, a FC média durante um jogo pode fornecer uma estimativa razoável da energia gasta $[23,24]$. Rupčić et al. [22] determinaram a relação entre a FC e a captação de oxigênio para estimar o VO2 de árbitros de basquetebol durante os jogos da 1a Liga do basquete croata. De fato, os autores utilizaram um teste laboratorial incluindo um protocolo de esteira (ie, método espiro-ergométrico) e relataram um VO2max médio de 52,5 $\pm 5,8 \mathrm{ml} . \mathrm{kg}$ - 1 . min-1, enquanto o GE médio foi de 14,03 $\pm 2,03 \mathrm{kcal}$.min-1 e $13,66 \pm 1,87 \mathrm{kcal}$.min -1 durante a $1^{\mathrm{a}}$ e a $2^{\mathrm{a}}$ metade do jogo, respectivamente. Embora 
a GE esteja diminuindo gradualmente à medida que o jogo chega ao fim, as comparações entre os períodos de jogo ( $Q$ e metades) não indicaram qualquer diferença significativa. Inversamente, Nabli et al. [7] mostraram uma diferença significativa ( $p<0,001)$ na GE de juízes de basquetebol entre 113,46 $\pm 18,16 \mathrm{kcal}$ no $1^{\circ} \mathrm{Q}$ e $137 \pm 27,5 \mathrm{kcal}$ no $4^{\circ} \mathrm{Q}$ durante os jogos do $\mathrm{U}-19$ que envolveram o sistema dos dois árbitros. No entanto, os jogos analisados por Rupčić et al. [22] foram disputados pelo sistema de três árbitros, o que pode explicar a controvérsia observada entre as conclusões desses estudos. Portanto, estudos sobre GE na arbitragem de basquete mostraram maiores demandas energéticas durante os jogos que envolvem o sistema de dois árbitros, especialmente no final do jogo, provavelmente por causa dos esforços dos árbitros para acompanhar o jogo. Além disso, Nabli et al. [7] expressaram os dados de GE dos árbitros em MET, usando equações específicas, a partir do tempo gasto pelo árbitro em cada zona de intensidade de exercício para determinar a intensidade metabólica das atividades dos árbitros durante os jogos. Este estudo mostrou que o GE médio ( 5 kcal.kg-1.h-1) correspondeu a "intensidade energética moderada" ( 5 METs) com uma grande contribuição da via de energia aeróbica. Durante $88 \%$ do tempo total do jogo, a intensidade foi igual ou inferior a 3,8 METs. No entanto, $12 \%$ do tempo total do jogo, a intensidade foi superior a 9,8 METs, que foram considerados como "baixos" e "alta intensidade", respectivamente [7].

Nabli et al. [2] relataram valores médios de concentração de lactato sanguíneo [La-] de 4,3 $\pm 3,9$ e $6,7 \pm 4,9$ mmol.L-1 no final da $1^{\text {a }}$ e da $2^{a}$ metade, respectivamente. A média relatada [La-] (5,5 $\pm 4,4 \mathrm{mmol}$.L-1) em Nabli et al. [2] estudo foi superior aos valores relatados por Borin et al [3], que encontraram os seguintes valores: qualificação $(2,1 \pm 0,8 \mathrm{mmol} . \mathrm{L}-1)$, semi-final $(2,7 \pm 0,9 \mathrm{mmol} . \mathrm{L}-1)$ e final fases $(2,9 \pm$ 0,8 mmol.L-1) em jogos masculinos seniores (com média de [La-] ao intervalo e no final do jogo). Isso pode ser atribuído à diferença no sistema de arbitragem durante os jogos analisados (U-19 vs. Jogos Seniores jogados com dois contra três árbitros, respectivamente). Embora estes os estudos não mostraram uma diferença significativa entre os tempos de medição [La-] (isto é, entre $Q$ e metades), os dados [La-] coletados de estudos de arbitragem de basquete mostram que o metabolismo anaeróbico é moderadamente solicitado. A esse respeito, foi relatado que o [La-] obtido durante jogos esportivos competitivos em equipe refletia principalmente a atividade realizada durante os últimos 5 minutos anteriores à coleta de sangue [25]. 
Além disso, foi relatado que a taxa de remoção ou depuração de lactato dependia da concentração de lactato sanguíneo, da atividade durante o período de recuperação e da capacidade aeróbia do participante [26]. Mudanças em [La-] também podem ser o resultado de estresse, ansiedade, pressão e responsabilidade dos árbitros no progresso do jogo. Sabe-se que o [La-] é afetado pelo sistema nervoso simpático, e foi demonstrado que o estresse psicossocial pode aumentar [La-] em até $47 \%$, o que tem sido atribuído ao aumento dos hormônios adrenocorticotrópicos [27].

\section{DEMANDA METABÓLICA DURANTE O JOGO}

A taxa de trabalho dos árbitros de basquetebol durante os jogos pode ser determinada pela análise de movimento usando procedimentos que foram adotados para monitorar jogadores (por exemplo, análises de tempo e movimento de vídeo) [28,29]. A distância total percorrida produz uma indicação indireta da GE [30], mas pode ser decomposta de acordo com a intensidade do movimento (ou seja, a distância e o tempo gasto em pé, andando, correndo, correndo, girando). Normalmente, é feita uma distinção entre atividades de intensidade baixa a moderada, como caminhar e correr, e atividades de alta intensidade, como corrida de velocidade. Tempos de inatividade quando o árbitro está parado podem também ser registrados. Análises de tempo e movimento em árbitros de basquete nas ligas brasileira, tunisiana e eslovena indicam que os árbitros cobrem entre 3.000 me $6500 \mathrm{~m}$ durante cada partida, independentemente do sistema de arbitragem aplicado durante as competições. Os maiores valores médios registados foram $6773 \mathrm{~m}$ na Liga eslovena [27] e $6450 \mathrm{~m}$ na Liga Brasileira [3], onde os jogos eram jogados pelo sistema de dois árbitros diferentes (dois e três árbitros por jogo, respectivamente). Quando as distâncias do sistema dos dois árbitros foram comparadas, mostrou que os árbitros do campeonato esloveno realizaram uma distância coberta média maior do que os árbitros da liga portuguesa e tunisiana durante os jogos oficiais. (6773 vs 4960 e 3907 m, respectivamente). Em relação ao jogo envolvendo o sistema dos três árbitros, Borin et al [3] relataram que a distância percorrida pelos árbitros de basquetebol brasileiros na fase de classificação foi significativamente menor do que nas semifinais e finais $(p<0,05)$. Isso sugere que $o$ nível da competição pode afetar a atividade física do árbitro independentemente do sistema de arbitragem usado durante os jogos oficiais [3]. 
Os árbitros de basquetebol gastam significativamente mais tempo em atividades de baixa intensidade e menos tempo envolvido em um sprint durante o jogo, ao comparar o tempo total de jogo [16,31]. A este respeito, os árbitros eslovenos cobriram uma distância total média de $5291 \mathrm{~m}$ durante os jogos liderados por três árbitros; eles

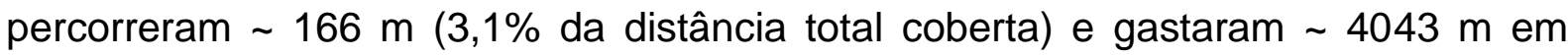
atividades de baixa intensidade (ou seja, <10,8 km.h-1) $(76,4 \%$ da distância total coberta). No entanto, um árbitro em uma tripulação de dois árbitros cobriu uma distância total média de 6773 m; eles percorreram 368 m (5,4\% da distância total percorrida) e gastaram $\sim 4536 \mathrm{~m}$ em atividades de baixa intensidade que representam $66,9 \%$ da distância total percorrida [31]. Parece que na tripulação de dois árbitros, as exigências aeróbicas e anaeróbicas entre os árbitros de basquetebol são maiores do que na tripulação de três árbitros, sabendo que os jogos foram disputados no mesmo nível e fase da competição. Por outro lado, os árbitros sub-19 da Tunísia cobriram $3907 \pm 848 \mathrm{~m}$ como distância total média durante os jogos, gastaram $81 \%$ do tempo total de jogo e $2 \%$ de velocidade de sprint [2]. Isso sugere que o nível de arbitragem do basquetebol na Tunísia era menor do que o nível europeu, pelo menos a partir de uma perspectiva de tempo-movimento.

Nabli et al. [2] (2016) relataram que atividades de baixa e moderada intensidade $(<10,11 \mathrm{~km} . \mathrm{h}-1)$ predominam durante os jogos (ou seja, $88 \%$ do tempo total), enquanto atividades de alta intensidade são usadas principalmente como um indicador de atividade física. desempenho [32]. Inconsistências na classificação de zonas de atividade para determinar corridas de alta intensidade tornaram as comparações entre estudos difíceis [8]. Na arbitragem do basquete, os limiares de alta intensidade da literatura variam de 14,83 a 18,72 km.h-1 [2,31] e, consequentemente, os árbitros de corrida de alta intensidade, como porcentagem da distância total do jogo, variam de $2 \%$ a $5,4 \%$. Alguns sugeriram o uso de limiares de velocidade arbitrários para individualizar as atividades de jogo em relação às velocidades de movimento específicas de cada árbitro ou capacidade fisiológica, mas isso merece futuras investigações [33].

A análise dos perfis de atividade dentro dos jogos geralmente é usada para examinar a possível aparência de fadiga durante o jogo. Acredita-se que as mudanças no perfil entre as atividades de alta intensidade sejam representativas da fadiga acumulada [8]. 
Os árbitros de basquetebol sub-19 estudados por Nabli et al. [2] gastaram menos distância percorrendo na $2^{\underline{a}}$ metade em comparação com a $1^{\text {a }}$ parte e diferenças significativas foram observadas em atividades de baixa intensidade, como caminhada e pé. De fato, os árbitros gastaram significativamente mais tempo em caminhada ( $p=$ $0,009)$ e em pé $(p<0,001)$ durante o 20 tempo. Especificamente, os árbitros gastaram significativamente mais tempo em atividades de baixa intensidade (pé e caminhada) com tamanho de efeito moderado (diferença acima de 21,86\%; $d=0,79 ; p=0,038$ e $\sim 5,24 \% ; d=1,11 ; p=0,0007$, respectivamente) no quarto $Q$ em comparação com o $1^{\circ} \mathrm{Q}$. No entanto, o tempo gasto em sprinting diminuiu no $2^{\circ}$ tempo em comparação com o $1^{\circ}$, o que representa um declínio no desempenho de alta intensidade dos árbitros no final do jogo. Isso pode ser devido à fadiga acumulada nos árbitros de basquete durante o segundo tempo do jogo. $\mathrm{Na}$ arbitragem de futebol, Reilly e Gregson [34] relataram que essa fadiga pode estar ligada à queda no ritmo do jogo associado com a queda concomitante na taxa de trabalho dos jogadores. De fato, jogadores de basquete tunisianos de elite sub-19 gastaram significativamente mais tempo em atividades de baixa intensidade ( $31,20 \pm 2,24$ vs. $32,61 \pm 1,23 \%$ do tempo total, $p<0,05)$ e menos tempo com corrida e corrida de alta velocidade ( $3,53 \pm 0,97$ vs. $2,13 \pm 0,46 \%$ do tempo total, $p<0,01)$ no $2^{\circ}$ tempo em relação ao $1^{\circ}$ tempo, respectivamente [28]. Além disso, nos jogos de basquete, as estratégias táticas poderiam explicar qualquer tendência de declínio na atividade de alta intensidade (sprint) no $4^{\circ} \mathrm{Q}$. De fato, a taxa de "reprodução direta" e quebra rápida diminui com o tempo, causando todo o tempo de execução. para abrandar [35].

Numerosos autores relataram muitas considerações metodológicas que influenciam a interpretação do desempenho físico dos árbitros em jogos de basquete. De fato, Borin et al. [3] relataram que as distâncias totais cobertas pelos árbitros no basquete parecem depender do nível de competição, especialmente entre as fases da competição. De fato, a distância total percorrida durante o jogo aumentou durante as fases de competição: 4020m, 4970m e 6170m para as fases de qualificação, semifinal e final dos jogos da liga de basquete, respectivamente). Independentemente do número de árbitros envolvidos durante os jogos, as exigências físicas impostas aos árbitros são melhor compreendidas quando a distância total é dividida em categorias de jogos relacionadas à velocidade [36]. Mais recentemente, Nabli et al. [2] forneceram estimativas mais precisas dos perfis de atividade de jogo de árbitros de RC: 34836

Disponível em: https://www.nucleodoconhecimento.com.br/educacao-fisica/estresse-fisiologico 
basquete, onde as atividades de jogo foram consideradas como tempo gasto e distância percorrida em cinco atividades de corrida (pé 0-2,92 km.h-1, caminhada> 2,92-7,34 km. h-1, corrida> 7.34-10.11 km.h-1, corrida> 10.11-14.83 km.h-1 e sprint> $14.83 \mathrm{~km} . \mathrm{h}-1)$. Os autores relataram uma média total da distância total do jogo de $3907 \pm 848 \mathrm{~m}$, com 926,65 \pm 369,5 m correndo e 485,65 $\pm 185,82 \mathrm{~m}$ de corrida. Isso foi mais baixo do que o relatado na $1^{\text {a }}$ liga eslovena de basquete (play-offs na temporada 2002/2003) [31], sabendo que a análise de tempo-movimento foi baseada em quatro categorias movimentos (caminhada <3,6 km.h-1, corrida lenta 3,6-10,8 km.h-1, corrida média 10,8-18 km.h-1 e corrida rápida> 18 km.h-1) e estilo de arbitragem (dois sistema de arbitragem por jogo) foi o mesmo. Mas esta diferença pode dever-se ao nível de competição (sub-19 vs. jogos seniores, respectivamente) e / ou ao nível de arbitragem (árbitros nacionais vs. internacionais). Esses dados reforçam a afirmação de que a arbitragem do basquete em tal nível representa um desafio físico bastante significativo. Este desafio exigente pode ser considerado semelhante ou até maior do que jogar basquete. De fato, os árbitros são em média 10 a 15 anos mais velhos que os jogadores. Esta comparação foi baseada em dados de árbitros e jogadores reunidos durante o campeonato de basquetebol sub-19 da Tunísia (35,7 $\pm 9,1$ vs 18,2 $\pm 0,5$ anos, respectivamente) [2,28].

\section{ENVELHECIMENTO E DESEMPENHO FÍSICO NO JOGO}

$\mathrm{Na}$ arbitragem de basquete, a NBA não aplicou qualquer idade de aposentadoria por muitos anos. Por exemplo, o árbitro "Dick Bavetta" na NBA aposentou-se aos 74 anos de idade, com quase 40 anos de serviço na arbitragem da NBA [37]. No entanto, a FIBA considerou recentemente a idade de cinquenta e um como um máximo para um Árbitro ativo [38]. A justificativa para a idade de aposentadoria não é clara, especialmente porque a prova recente sugere que o declínio da aptidão aeróbica relacionado à idade (ou seja, VO2max) não é tão esperado [19]. Enquanto valiosos insights sobre o efeito do envelhecimento no perfil antropológico do árbitro de basquete foram relatados [19]; a relação entre idade e desempenho físico / fisiológico nos jogos não foi relatada.

Nesse contexto, Rupčić et al. [19] compararam os resultados morfológicos, fisiológicos, físicos, de "qualidade de arbitragem" e "experiência" entre árbitros de 
basquetebol jovens (26-32 anos) e idosos (33-46 anos). Eles relataram uma diferença estatisticamente significativa $(p<0,01)$ em todos os testes físicos para confirmar que as habilidades físicas dos árbitros diminuem gradualmente com a idade cronológica. Além do que, além do mais, os resultados mostraram como os árbitros foram estatisticamente diferentes em "Qualidade de arbitragem" e "Experiência". De fato, os árbitros "mais velhos" mostraram uma pontuação mais alta na qualidade de arbitragem em comparação aos árbitros de basquete "mais jovens" [19]. Além disso, os árbitros "mais velhos" tinham, obviamente, muito mais experiência (10,6 anos) em arbitragem ao mais alto nível de competição em comparação com os árbitros "mais jovens" (4,2 anos). Mas não houve diferença estatisticamente significante no nível das capacidades fisiológicas dos árbitros de basquetebol de acordo com sua idade cronológica (capacidade aeróbia: VO2max foi de 54,04 vs. 50,84 ml.kg-1.min-1 para árbitros jovens e mais velhos, respectivamente). ). Portanto, atenção especial deve ser dada ao treinamento físico de árbitros mais velhos para garantir que eles possam continuar atendendo às demandas físicas do jogo. A este respeito, Rupčić et al. [19] mostraram que a experiência é um fator crucial indispensável para a prática de jogos de basquete com sucesso. De fato, os árbitros experientes têm um conhecimento teórico enriquecido, porque as horas acumuladas nos jogos oficiais levam a adaptações específicas do papel e à melhoria de estruturas e processos de conhecimento que suportam a codificação e a recuperação de informações da memória [39]. É um fato natural que as habilidades físicas de árbitros de basquete cronologicamente mais velhos diminuam gradualmente [19], mas considerando a complexidade do jogo, elas compensam essa deficiência relativa com sua maior experiência arbitral [19].

\section{FUNÇÃO COGNITIVA E FADIGA}

O árbitro é obrigado a interpretar as regras do jogo e garantir que a jogada possa prosseguir de maneira imparcial e segura [40]. Embora tais demandas pareçam simples, o papel é extremamente dificultado pelo volume total de decisões que devem ser tomadas, a natureza subjetiva de muitas dessas decisões e a influência de variáveis contextuais [8]. De fato, os árbitros de basquete devem tomar decisões rápidas e complexas enquanto estiverem sob estresse e pressão (por exemplo, barulho de torcida, pressão exercida pelos jogadores e treinadores, movimento 
contínuo dos jogadores, tornando a visão da infração não fácil de seguir: quando um jogador mascara outro jogador, por exemplo). Portanto, escolhas "são feitas sob pressão, onde o ruído de uma multidão excitada poderia exercer e criar uma" vantagem em casa ", o que poderia influenciar as decisões do árbitro e, portanto, o resultado do jogo" [34]. Estudos recentes sugerem que os níveis de alcance das vítimas flutuam nos jogos, dependendo do diferencial de pontuação e do tempo de jogo (ou seja, mais pressão à medida que o tempo expira) [41]. Além disso, a análise das chamadas dos árbitros em 250 desses incidentes, tirada de uma temporada inteira da Superliga de Basquetebol de Israel, concluiu que a reputação dos jogadores e das equipas, a questão das equipas em casa e fora de casa e os jogadores 'tamanho físico não afetou significativamente a frequência de chamadas para uma falta ofensiva [42].

Parâmetros internos como a fadiga fisiológica também podem afetar as habilidades dos árbitros e suas funções cognitivas. Isso pode ser mais crítico no final do jogo, porque as estatísticas informaram que a maioria dos jogos de basquete em qualquer nível competidor termina com um a três pontos a favor da equipe vencedora [19]. Infelizmente, os efeitos da última fadiga na função cognitiva dos árbitros de basquete e, portanto, potencialmente, suas capacidades de decisão não foram estudados até o momento.

Outra questão fundamental no basquete é a relação entre fadiga fisiológica e desempenho cognitivo, que parecia fornecer resultados um pouco contraditórios na literatura. Os vários resultados são difíceis de comparar, porque os estudos testaram participantes de diferentes origens e com diferentes níveis de aptidão, e várias tarefas cognitivas foram usadas durante ou após tarefas de exercícios de durações diversificadas [43]. Muitas das evidências da literatura sugerem que a relação entre a intensidade da atividade física aguda e o desempenho cognitivo tem uma forma em $U$ invertida [44]. A esse respeito, o desempenho cognitivo dos árbitros (isto é, tomada de decisão e reação tempo) pode ser prejudicada por sua fadiga fisiológica. Cian et al. [45] relataram que durante exercício físico submáximo prolongado (isto é, corrida de duas horas em esteira com $65 \%$ VO2max) ocorreu redução no desempenho cognitivo, como interrupção da memória de curto prazo, habilidades psicomotoras e discriminação visual. (observe que a desidratação foi um fator de confusão em relação a essas observações). Além disso, o exercício fatigante (por exemplo, correr acima 
do limiar anaeróbico) com carga de trabalho pesada impedia especificamente as habilidades mentais superiores, como o desempenho da tomada de decisão [43]. De fato, a fadiga física afeta principalmente o funcionamento cognitivo complexo que requer um grande processo de recursos do sistema nervoso central [46]. No entanto, vários estudos descobriram que, imediatamente após uma sessão de exercício de intensidade submáxima (isto é, FC de cerca de 110-130 bat.min-1) com uma duração de 20-40 min, houve uma melhora no desempenho cognitivo [44,47] . De fato, tem sido sugerido que o exercício de intensidade moderada provocou evidências de ativação neuroelétrica superior, que fornece o nível ideal de excitação e, como consequência, tem o efeito mais benéfico sobre os recursos atencionais necessários para o desempenho cognitivo [48]. Embora algumas controvérsias tenham sido relatadas, foi demonstrado que existe uma relação entre a intensidade e a duração da atividade física e da função cognitiva [44]. A influência da fadiga na função cognitiva ainda é assunto de debate. De fato, é incerto se a fadiga fisiológica acarreta um declínio no desempenho mental relacionado ao jogo e não se sabe se a função executiva do cérebro está relacionada à tensão fisiológica e às deteriorações de desempenho experimentadas pelos árbitros. Assumimos que qualquer deterioração na tomada de decisões ocorreria quando o metabolismo cerebral fosse afetado, mas as avaliações existentes baseadas em laboratório da função cognitiva não são suficientemente sensíveis para detectar esse declínio [34]. Enquanto a reidratação e a provisão de energia ajudariam a atrasar e compensar a fadiga muscular, seus efeitos sobre os "fatores centrais" dos árbitros no jogo ainda não estão estabelecidos [34].

Existe uma clara necessidade de pesquisa que identifique os mecanismos de tomada de decisão em árbitros de basquete. Como os árbitros extraem informações relevantes das situações do jogo para construir seus julgamentos? Quais são as estratégias e heurísticas (ou seja, abordagem ou método usado para reduzir os julgamentos complexos aos mais simples) que os árbitros de basquete envolvem ao fazer julgamentos? Como esses processos são influenciados por fatores internos (por exemplo, fadiga) e externos ao árbitro? Para que a ciência tenha um impacto positivo no desenvolvimento de árbitros, é necessário um melhor entendimento sobre os fatores que diferenciam os árbitros de elite dos de sub-elite e como as intervenções de treinamento podem ser desenvolvidas para facilitar a aquisição mais rápida das habilidades subjacentes ao melhor desempenho. 


\section{AVALIAÇÃO DE APTIDÃO DE ÁRBITROS}

Os alvos de aptidão para árbitros foram baseados em critérios de desempenho em vez de avaliação fisiológica [34]. A aptidão dos árbitros de elite é considerada de fundamental importância para o sucesso do arbitro [36]. A esse respeito, o teste de campo tem sido incluído há muito tempo como parte dos critérios de seleção de arbitragem pelos órgãos diretivos nacionais e internacionais de arbitragem.

A avaliação da resistência dos árbitros de basquetebol consiste na estimativa da capacidade aeróbica (VO2max) através da corrida de 20 m com o nível mínimo de aptidão (isto é, alvo a ser considerado apto) arbitrariamente nomeado sem base científica [4]. Padrões para alcançar a acreditação a nível nacional e internacional foram sucessivamente levantados para melhorar a aptidão. A menos que os árbitros mantenham seu nível de desempenho nesses testes, sua acreditação é retirada [34]. O árbitro da FIBA t este de aptidão específico é uma corrida de $20 \mathrm{~m}$. O teste é realizado em uma quadra de basquete; os participantes são obrigados a correr entre os marcadores separados por $20 \mathrm{~m}$ enquanto guiados pelo padrão de bips sonoros. Os participantes masculinos e femininos devem correr, seguindo estritamente o sinal, $1720 \mathrm{~m}$ (86 voltas $\times 20 \mathrm{~m}$ ) e $1320 \mathrm{~m}$ (66 voltas $\times 20 \mathrm{~m}$ ), respectivamente [48].

Ao considerar as demandas específicas de jogo impostas aos árbitros de basquete, o teste de corrida de 20 metros pode ser criticado por falta de validade de conteúdo, em que os árbitros nunca realizam corridas consecutivas de 2 a $20 \mathrm{~m}$ enquanto estiverem arbitrando. Por outro lado, a validade é a medida em que um teste mede o que é projetado para medir e um teste físico possui boa validade de conteúdo se representar adequadamente o domínio da tarefa física [51]. Em uma tentativa de verificar a relação entre o desempenho do jogo dos árbitros; respostas fisiológicas e o desempenho do teste de recuperação intermitente YO-YO nível 1 (YO-YO-IRT1), Nabli et al. [2] descobriram que o desempenho de YO-YO-IRT1 foi significativamente correlacionado ( $p<0,05)$ com: a) distância percorrida em atividades de baixa intensidade (ie caminhada e jogging) eb) intensidade de jogo (ie\% FCmáx) em períodos de jogo . Isto pode indicar a validade direta deste teste intermitente de resistência de alta intensidade (isto é, YO-YO-IRT1) para avaliar as capacidades aeróbicas dos árbitros de basquetebol. Dado que o YO-YO -IRT1 é atualmente o único teste de aptidão para 
ter validade direta; seu uso deve ser considerado pelos órgãos diretivos (ou seja, a comissão técnica da FIBA) para a avaliação dos árbitros de basquete. O YO-YO-IRT1 pode refletir mais especificamente o perfil de exercício intermitente da jogabilidade e da atividade do árbitro.

Capacidade de sprint repetida (RSA) é uma importante qualidade física que discrimina desempenho em jogadores de basquetebol [52] e, portanto, sua pertinência para árbitros é inteligível, como suas atividades físicas podem ser orientadas por aqueles dos jogadores [2].

Embora a FIBA não tenha proposto um teste RSA específico para árbitros de basquete, uma investigação inicial [16] relatou que o índice de fadiga do teste RSA estava relacionado à distância percorrida em atividades de alta intensidade por árbitros durante a $2^{\mathrm{a}}$ metade e $3^{\mathrm{a}}$. Jogos de basquete sub-19 $(r=-0,54, p=0,029 ; r=$ $-0,5, p=0,047$, respectivamente). Deve-se levar em consideração que Nabli et al. [2] selecionaram um protocolo RSA específico usado em uma repetição de sprints com uma mudança acentuada de direção $\left(180^{\circ}\right)$ em uma curta distância $(2 \times 15 \mathrm{~m})$ com $25 \mathrm{~s}$ de recuperação passiva no meio. Esse resultado das correlações observadas pode ser devido a um aumento da ocorrência de fadiga acumulada em árbitros de basquete repetindo atividades de alta intensidade no final do jogo. No entanto, o decréscimo no desempenho do sprint do árbitro pode ser afetado por vários fatores, independentemente do esporte. No futebol, um declínio no desempenho na segunda metade do jogo foi associado a uma diminuição dos estoques de glicogênio muscular [53]. No basquete, as estratégias táticas também podem explicar as mudanças nas atividades de alta intensidade nos últimos minutos (ou seja, $4^{\circ} \mathrm{Q}$ ) do jogo; onde as equipes tendem a gerenciar e controlar a posse de bola, a fim de impedir que o time perdedor tenha ocasiões de marcar pontos para compensar a pontuação. Consequentemente, a proporção de jogadas retas e rápidas é provável que diminua, induzindo todo o ritmo a abrandar [24].

Mais estudos são necessários para fortalecer a confiabilidade e a validade dos testes (ou seja, RSA e YO-YO-IRT1), juntamente com uma robusta validação empírica dos padrões mínimos de desempenho para os árbitros de elite.

\section{CONCLUSÕES}


Por fim, apresentamos uma visão geral e perspectivas em potencial. Os árbitros de basquete exigem altos padrões de habilidades físicas e de funcionamento cognitivoperceptivo. Esses requisitos precisam de avaliação de desempenho e implementação subsequente de programas estruturados de treinamento que atendam às diferentes demandas de arbitragem de elite. Relatórios recentes demonstram que a performance física de árbitros de basquetebol em jogos é significativamente $(p<0,05)$ correlacionada com suas próprias habilidades físicas individuais. Um programa de treinamento usando distâncias mais curtas de sprint (20-30 m com mudança de direção), com menor tempo de recuperação entre os sprints (20-30 s), poderia melhorar o desempenho repetido dos sprints (ou seja, energia anaeróbica) durante os jogos. Um programa de treinamento intermitente incluindo a proporção de 1: 12 de "esforço / recuperação de alta intensidade" poderia ser prescrito para desenvolver a resistência intermitente de alta intensidade (ou seja, capacidade aeróbica) dos árbitros de basquete.

Os desempenhos físicos reduzidos em árbitros de basquetebol mais velhos não diminuíram significativamente $(p<0,05)$ a sua capacidade de manter o jogo, portanto; esses árbitros experientes parecem ser mais qualificados para oficiar. Um exame sobre se os árbitros mais velhos ainda são capazes de atender às demandas físicas e cognitivas de seus jogos pode ser determinado através da avaliação dos desempenhos técnicos e físicos do jogo, juntamente com o desempenho do treinamento e níveis de aptidão durante um período longitudinal.

Apesar de o teste YO-YO-IRT1 ser considerado recentemente como teste de aptidão específico entre os árbitros do FIBA Elite Group (desde junho de 2018 pela World Technical Commission), o FIBA National Competitions ainda usa o teste shuttle $20 \mathrm{~m}$ (teste Beep). O teste RSA ( $2 \times 15 \mathrm{~m}$ com $25 \mathrm{~s}$ de recuperação passiva no meio) deve ser considerado como um teste de aptidão específico para avaliar a capacidade de manter e repetir o sprint dentro de árbitros de basquete.

Todos os estudos acima mencionados nesta revisão analisaram as habilidades físicas (ou seja, análises e testes de tempo-movimento), fisiológicas, mentais ou de tomada de decisão e envelhecimento entre os árbitros de basquete, mas não há pesquisas 
que tiveram estudou todos eles juntos. Tais estudos seriam bem-vindos, provavelmente impactando significativamente nosso conhecimento no campo.

A presente revisão mostra que a arbitragem de basquetebol exige demandas específicas (isto é, parâmetros físicos, fisiológicos, perceptivos e cognitivos) para mantê-los com o jogo. A análise de movimento do tempo dos árbitros de basquete revelou uma atividade intermitente e a determinação da GE reflete um esforço de intensidade moderada durante os jogos. Além disso, o teste de aptidão deve basearse em critérios válidos e escolhas de testes (ou seja, YO-YO-IRT1 para avaliar a atividade intermitente e capacidade aeróbica), para oficiar o jogo de basquete. Os programas de treinamento devem ser baseados nas atividades de análise de jogos dos árbitros de basquete. Para permitir que os árbitros lidem com a fadiga física / mental durante os jogos oficiais, os programas de treinamento devem levar em consideração: (a) a idade dos árbitros (início ou fim da carreira) e o nível de condicionamento físico; (b) a experiência ou padrão dos árbitros (nacional ou internacional, sistema de dois ou três árbitros) e (c) o nível de competição (masculino ou feminino, sênior ou jogos juvenis).

\section{REFERÊNCIAS}

1. National Basketball Association Official Rules 2009-2010. Rule 2, Section I, a. Available http://i.cdn.turner.com/nba/nba/.element/pdf/2.0/sect/officiating/Official_NBA_ R ule_Rook_09-10.pdf. [Last accessed 10 September 2018].

2. Nabli MA, Ben Abdelkrim N, Castagna $C$, et al. Physical and physiological demands of U-19 basketball refereeing: Aerobic and anaerobic demands. The Physician and Sports Medicine. 2016;44(2):158-63.

3. Borin JP, Daniel JF, Bonganha V, et al. The distances covered by basketball referees in a match increase throughout the competition phases, with no change in physiological demand. Journal of Sports Medicine. 2013;4:193-198.

4. Leicht AS. Physiological demands of basketball refereeing during international competition. Journal of Science and Medicine in Sport. 2008;11:357-360. 
5. Vaquera A, Mielgo-Ayuso J, Calleja-González J, et al. Cardiovascular and perceptual stress of female basketball referees during women's International matches. Journal of Sports Medicine and Physical Fitness. 2016;57(4):476-482.

6. Vaquera A, Mielgo-Ayuso J, Calleja-González J, et al. Sex differences in cardiovascular demands of refereeing during international basketball competition. The Physician and Sports Medicine. 2016;11:1-6.

7. Nabli MA, Ben Abdelkrim N, Castagna C, et al. Energy demands and metabolic equivalents (METS) in U-19 basketball refereeing during official games. Journal of Sports Medicine \& Doping Studies. 2017;7:190.

8. Weston M, Castagna C, Impellizzeri FM, et al. Science and medicine applied to soccer refereeing an update. Sports Medicine. 2012;42(7):615-631.

9. Matković A, Rupčić T, Knjaz D. Physiological load of referee during basketball games. Kinesiology. 2014;(46)2:258-265.

10. Vaquera A, Mielgo-Ayuso J, Calleja-González J, et al. Match intensity and heart rate predictors in top level basketball referees during men's Eurobasket. J Sports Med Phys Fitness. 2016;56(9):1034-40.

11. Cestaro Júnior $S$, Molina R, Denadai BS. Análise do deslocamento do árbitro em quadra no primeiro e segundo período da partida de basquetebol. [Analysis of the referee's displacement on court in the first and second match periods]. Revista Treinamento Desportivo. 2001;6:31-37. Portuguese.

12. Vučković $G$, Dezman B. Results of tracking a referee's movements during a basketball match with computer sight. In: JÜRIMÄE, Toivo (ed.). Sport kinetics: human movement as a science in the new millenium: proceedings. Acta Kinesiologiae, Universitatis Tartuensis. 2001;6:274-277.

13. Galy O, Ben Zoubir S, Hambli M, et al. Relationships between heart rate and physiological parameters of performance in top-level water polo players. Biology of Sport. $2014 ; 31(1): 33-38$.

14. Leicht AS. Cardiovascular stress on an elite basketball referee during national competition. British Journal of Sports Medicine. 2004;38(4):E10.

15. Leicht AS. Aerobic power and anthropometric characteristics of elite basketball referees. J Sports Med Phys Fitness. 2007;47(1):46-50. 
16. Nabli MA, Abdelkrim N, Jabri I, et al. Fitness field tests' correlation with game performance in U-19-Category Basketball referees. International Journal of Sports Physiology and Performance. 2016;11(8):1005-1011.

17. Catterall C, Reilly T, Atkinson G, et al. Analysis of the work rates and heart rates of association football referees. British Journal of Sports Medicine. 1993;27:193- 196.

18. ACSM. American College of Sports Medicine Position Stand. The recommended quantity and quality of exercise for developing and maintaining cardiorespiratory and muscular fitness, and flexibility in healthy adults. Med Sci Sports Exerc. 1998;30: 97591.

19. Rupčić T, Matković BR, Knjaz D, et al. Differences in the anthropological profile of the basketball referees with regards to their chronological age. Sport Logia. $2011 ; 7(1): 27-33$.

20. Heimer S, Mišigoj-Duraković M, Ružić L, et al. Fitness level of adult economically active population in the republic of Croatia estimated by EUROFIT system. Collegium Antropologicum. 2004;28(1):223-233.

21. Bonganha V, cavaglieri CR, Daniel JF, et al. Neuromuscular and metabolic characteristics of elite basketball referees. J Sports Med Phys Fitness. 2013;53:217-23.

22. Rupčić T, Matković BR, Knjaz D, et al. Differences in physiological load of the referees with consideration to the period of the basketball game. Sport Logia. 2012;8(1):51-56.

23. Bangsbo J. The physiology of soccer with special reference to intense intermittent exercise. Acta Physiologica Scandinavica. 1994;151(619):1-156.

24. Reilly, T. Energetics of high intensity exercise (soccer): With particular reference to fatigue. Journal of Sports Sciences. 1997;15(3):257-63.

25. Gilman MB. The use of heart rate to monitor the intensity of endurance training. Sports Medicine. 1996;21:73-9. 
26. Stølen T, Chamari K, Castagna C, et al. Physiology of Soccer an update. Sports Medicine. 2005;35(6):501-536.

27. Kubera B, Hubold C, Otte S, et al. Rise in plasma lactate concentrations with psychosocial stress: A possible sign of cerebral energy demand. Obes Facts. 2012;5(3):384-392.

28. Ben Abdlekrim N, El Fazaa S, El Ati J. Time-motion analysis and physiological data of elite under- 19 Basketball players during competition. British Journal of Sports Medicine. 2007;41(2):69-75.

29. Scanlan AT, Dascombe BJ, Kidcaff AP, et al. Gender-Specific activity demands experienced during semiprofessional basketball game play. International Journal of Sports Physiology and Performance. 2015;10(5):618-25.

30. Reilly T, Thomas V. Estimated daily energy expenditures of professional association footballers. Ergonomics. 1979;22:541-548.

31. Lončar M, Dežman B, Licen S. Tracking two and three officials with a computer. FIBA Assist Magazine. 2004;20:40-43.

32. Krustrup P, Bangsbo J. Physiological demands of top-class soccer refereeing in relation to physical capacity: Effect of intense intermittent exercise training. Journal of Sports Sciences. 2001;19:881-891.

33. Weston M, Drust B, Gregson W. Intensities of exercise during match-play in FA Premier League referees and players. Journal of Sports Sciences. 2011;29:527-32.

34. Reilly T, Gregson W. Special populations: The referee and assistant referee. Journal of Sports Sciences. 2006;2:795-801.

35. Ben Abdlekrim N, Castagna C, Jabri I, et al. Activity profile and physiological requirements of junior elite basketball players in relation to aerobic-anaerobic fitness. Journal of Strength and Conditioning Research. 2010;24(9):2330-2342.

36. Castagna C, Abt G, D'Ottavio S. Physiological aspects of soccer refereeing performance and training. Sports Medicine. 2007;37:625-46. 
37. Zach H. Report: Longtime NBA referee Dick Bavetta to retire at 74. 2014; Available at: http://www.cbssports.com/nba/eye-on-basketball/24644626/report- longtime-nbareferee-dick-bavetta-to-retire-at-74. [Last accessed 10 September 2018].

38. FIBA officials licensing 2017+. Operations Manual for national member federations. Periods 2017-2019 [PDF document]: Available at: http://www.fiba.basketball/en/Module/c9dad82f-01af-45e0-bb85ee4cf50235b4/9cf4fb76-856a-4d04-85e7-1f777ce065a3 [Last accessed August 12 2017].

39. Ericsson KA, Kintsch W. Long-term working memory. Psychological Review. 1995;102:211-45.

40. Fuller CW, Junge A, Dvorak J. An assessment of football referees' decisions in incidents leading to player injuries. American Journal of Sports Medicine. 2004;32(1):17-22S.

41. Ritchie J, Basevitch I, Rodenberg R, Tenenbaum G. Situation criticality and basketball officials' stress levels. J Sports Sci. 2017 Nov;35(21):2080-2087.

42. Morgulev E, Azar OH, Lidor R, Sabag E, Bar-Eli M. Searching for Judgment Biases Among Elite Basketball Referees. Front Psychol. 2018 Dec 20;9:2637.

43. Hogervorst E, Riedel W, Jeukendrup A, et al. Cognitive performance after strenuous physical exercise. Percept Motor Skills. 1996;83:479-88.

44. Abd-Elfattah, H. M., Abdelazeim, F. H., \& Elshennawy, S. (2015). Physical and cognitive consequences of fatigue: A review. Journal of Advanced Research, 6, 351358.

45. Cian C, Barraud PA, Melin B, et al. Effects of fluid ingestion on cognitive function after heat stress or exercise induced dehydration. International Journal of Psychophysiology. 2001;42:243-51.

46. Fleury M, Bard C, Carrière L. The effects of physical or perceptual work loads on a coincidence/anticipation task. Perceptual and Motor Skills. 1981;53(3):843-50. 
47. Chang YK, Chi L, Etnier JL, et al. Effect of acute aerobic exercise on cognitive performance: Role of cardiovascular fitness. Psychology of Sport and Exercise. 2014; 15(5):464-470.

48. Kamijo $\mathrm{Y}$, Nishihira A, Hatta $\mathrm{T}$, et al. Changes in arousal level by differential exercise intensity. Clinical Neurophysiology. 2004;115:2693-2698.

49. Léger LA, Mercier D, Gadoury C, et al. The multistage 20 meter shuttle run test for aerobic fitness. Journal of Sports Sciences. 1988;6:93-101.

50.

FIBA

Americas

Officiating.Available

at:

https://fibaamericasofficiating.com/2017/01/08/physical-preparation. [Last accessed 10 October 2018].

51. Payne W, Harvey J. A framework for the design and development of physical employment tests and standards. Ergonomics. 2010;53:858-71.

52. Attene G, Laffaye G, Chaouachi A, et al. Repeated sprint ability in young basketball players: one vs. two changes of direction (Part 2). Journal of Sports Sciences. 2015;33(15):1553-63.

53. Saltin B. Metabolic fundamentals in exercise. Medicine and Science in Sports. 1973;5:137-146.

Enviado: Junho, 2019.

Aprovado: Julho, 2019. 